\title{
Alguns dados sôbre a dinâmica populacional de uma localidade do interior da Amazônia
}

\author{
VANia FonseCa ( $*$ ) \\ Cleusa Marta CORrÊa (*) \\ Instituto Nacional de Pesquisas \\ da Amazônia
}

\begin{abstract}
SINOPSE
Os dados apresentados neste trabalho foram co. letados para o estudo que tem por objetivo levantar as principais características populacionais de agrupamentos humanos do interior da Amazônia. Embora êsses dados representem apenas $5 \%$ da amostra pretendida, são apresentados separadamente por terem sido coletados em uma comunidade militar, enquanto que tôdas as outras comunidades da amostra são formadas por população civil. Os autores se limitam, por ora, a apresentar os principais dados processados, deixando a análise e conclusões, para quando tiver sido completada a amostra.
\end{abstract}

Os dados apresentados neste trabalho compõem uma parte dos dados para o estudo que tem por objetivo levantar as principais características populacionais de agrupamentos humanos do interior da Amazônia.

Êsses dados foram colhidos em uma comunidade militar do interior do Estado do Amazo- nas e sua apresentação em separado prende-se a êsse motivo, uma vez que tôdas as outras comunidades pesquisadas são compostas bàsicamente por população civil.

Como êsse material representa menos de $5 \%$ da amostra pretendida, 110000 dados colhidos através de 2000 questionários, limitamo-nos a expor os principais dados processados, deixando a apresentação da análise e conclusões para quando tivermos completado a amostra.

Procuramos levantar informações que possibilitassem o conhecimento da Dinâmica Populacional da comunidade, através da análise de dois de seus fatores determinantes: fertilidade e migrações. Não foi possivel obtermos os dados necessários para uma análise da mortalidade geral dessa população.

A aplicação de questionários para a coleta dos dados foi feita em 96 dos 171 domicílios de militares radicados na localidade pesquisada, em maio de 1971.

\section{A - FERTILIDADE E NUPCIALIDAdE}

TABELA I - Idade da mulher entrevistada e número de filhos que elá teve.

\begin{tabular}{|c|c|c|c|c|c|c|c|c|c|c|c|c|}
\hline \multirow{2}{*}{$\begin{array}{c}\text { IDADE } \\
\text { DA } \\
\text { MULHER }\end{array}$} & \multicolumn{11}{|c|}{ NUMERO DE FILHOS } & \multirow{2}{*}{ TOTAL } \\
\hline & 0 & 1 & 2 & 3 & 4 & 5 & 6 & 7 & 8 & 9 & $10+$ & \\
\hline 15 a 20 & 4 & 3 & 3 & 1 & - & - & - & - & - & - & -- & 11 \\
\hline 20 a 24 & 1 & 5 & 7 & 6 & 3 & 2 & 1 & - & - & - & - & 25 \\
\hline 25 a 29 & - & 3 & 3 & 4 & 2 & 2 & 3 & 1 & 1 & - & - & 19 \\
\hline 30 a 34 & - & 1 & 2 & 5 & 2 & 3 & 2 & 2 & 2 & - & 2 & 21 \\
\hline 35 a 39 & 1 & - & 1 & 3 & 1 & 2 & 1 & 1 & 1 & 1 & 1 & 13 \\
\hline 40 a 44 & - & - & - & - & - & - & - & 1 & - & - & 1 & 2 \\
\hline $45 e+$ & - & - & - & - & - & - & - & - & - & - & - & - \\
\hline Ignorada & - & - & 一 & - & - & - & - & - & - & - & - & 1 \\
\hline Total & 6 & 12 & 16 & 19 & 8 & 9 & 7 & 5 & 4 & 1 & 5 & 92 \\
\hline
\end{tabular}

(*) - Bolsistas do Conselho Nacional de Pesquisas. 
O processo de amostragem utilizado foi probalilístico, por etapa dupla: domicílio constituindo a unidade primária e mulher do chefe a unidade secundária.

Através da entrevista com a mulher do che fe, foram colhidas informações sôbre: fecundidade, fertilidade, nupcialidade, migração, mortalidade infantil, composição faniliar, estrutura por sexo e idade.
Nem sempre fci possível obtermos declarações exatas por parte das entrevistadas, seja por faita de lembrança, seja por relutância em fornecer a informação, Por êsse motivo, quatro questionários foram abandonados, sendo utilizados os 92 restantes. Os principais dados colhidos pela aplicação dêsses questionários são apresentados nas tabelas I a XV.

TABELA II - Idade da mulher entrevistada e número de filhos vivos que ela tem

\begin{tabular}{|c|c|c|c|c|c|c|c|c|c|c|c|c|}
\hline \multirow{2}{*}{$\begin{array}{l}\text { IDADE } \\
\text { DA } \\
\text { MULHER }\end{array}$} & \multicolumn{11}{|c|}{ NOMERO DE FILHOS } & \multirow{2}{*}{ TOTAL } \\
\hline & 0 & 1 & 2 & 3 & 4 & 5 & 6 & 7 & 8 & 9 & $10+$ & \\
\hline 15 a 20 & 4 & 4 & 2 & 1 & - & - & - & - & - & - & - & 11 \\
\hline 20 a 24 & 1 & 7 & 7 & 4 & 3 & 2 & 1 & - & - & - & - & 25 \\
\hline 25 a 29 & - & 4 & 4 & 4 & 1 & 3 & 1 & 1 & 1 & - & - & 19 \\
\hline 30 a 34 & 1 & - & 2 & 6 & 1 & 5 & 2 & 2 & - & - & 2 & 21 \\
\hline 35 a 39 & 1 & 1 & 1 & 2 & 1 & 3 & 1 & 1 & - & 1 & 1 & 13 \\
\hline 40 a 44 & - & - & - & - & - & 1 & - & - & 一 & - & 1 & 2 \\
\hline 45 e + & - & - & - & - & - & - & - & - & - & - & - & - \\
\hline Ignorada & - & - & - & - & - & - & - & - & 一 & - & 1 & 1 \\
\hline Total & 7 & 16 & 16 & 17 & 6 & 14 & 5 & 4 & 1 & 1 & 5 & 92 \\
\hline
\end{tabular}

TABELA III - Respostas às perguntas: A) Por que a senhora não gostaria de ter mais filhos ? e B) Por que a senhora não gostaria de ter menos filhos ? (As perguntas foram feitas baseadas no número ideal de filhos, declarado pela entrevistada).

RESPOSTAS À PERGUNTA “A” :

Devido a não poder dar aos filhos a educação que gostaria ................ 9

Porque dá muito trabalho criar filhos ..... 27

Porque os filhos dão muita preocupação .. 2

Porque é difícil dar educação e manter os

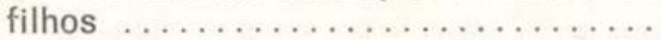
Porque sofre muito para ter o filho ...... 1

Porque o médico aconselhou ........... 5

Porque fica difícil viajar ............. 4

Não sabe; porque o ideal é êsse mesmo .. 5

Gostaria de ter muitos filhos, pois quanto

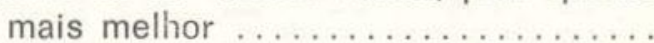

T o t a I
RESPOSTAS À PERGUNTA "B" :

Porque se tiver poucos filhos, êles podem morrer e não fica nenhum $\left({ }^{*}\right)$.......

Porque a casa fica sem animação e os filhos é que fazem companhia aos pais .... 6

Porque a família fica muito pequena ..... 41

Porque um filho faz companhia ao outro .. 1

Porque cêdo a mãe fica sozinha ....... 4

Nâu sabe; porque o ideal é êsse mesmo . 31

Não responde $\ldots \ldots \ldots \ldots \ldots \ldots \ldots \ldots$. 4

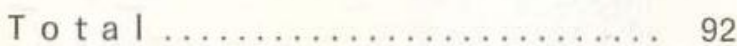

$(*)$ - Muitas entrevistadas, ao responderem a pergunta, cítavam o dito popular : "Quem tem dois tem um, quem tem un não tem nenhum". 
TABELA rv - Número de gestações e número de abortos sofridos pela mulher

\begin{tabular}{|c|c|c|c|c|c|c|c|c|c|c|c|c|}
\hline \multirow{2}{*}{$\begin{array}{l}\text { NUMERO } \\
\text { DE } \\
\text { ABORTOS }\end{array}$} & \multicolumn{11}{|c|}{ NUMERO DE GESTACOOES } & \multirow{2}{*}{ TOTAL } \\
\hline & 0 & 1 & 2 & 3 & 4 & 5 & 6 & 7 & 8 & 9 & $10+$ & \\
\hline $0 \ldots \ldots \ldots$ & 6 & 10 & 13 & 13 & 8 & 6 & 6 & 2 & 3 & 1 & 3 & 71 \\
\hline $1 \ldots \ldots \ldots$ & - & - & 1 & 3 & 3 & - & 3 & 1 & 1 & 3 & - & 15 \\
\hline $2 \ldots \ldots \ldots$ & - & - & - & 1 & 1 & - & - & - & - & - & 1 & 3 \\
\hline $3 \ldots \ldots \ldots$ & - & - & - & - & - & $一$ & 1 & - & - & - & - & 1 \\
\hline $4 \ldots \ldots \ldots$ & - & - & - & - & - & - & - & 1 & - & - & - & 1 \\
\hline $5 \ldots \ldots \ldots$ & - & - & - & - & - & - & - & - & - & - & 一 & - \\
\hline $6 \ldots \ldots \ldots$ & - & - & - & - & - & - & -- & - & - & - & 1 & 1 \\
\hline $7 e+\ldots$ & - & - & - & - & - & - & - & - & - & - & - & - \\
\hline Total .... & 6 & 10 & 14 & 17 & 12 & 6 & 10 & 4 & 4 & 4 & 5 & 92 \\
\hline
\end{tabular}

TABela $\mathrm{v}$ - Tipo de união e uso de meios anticoncepcionais (MAC)

\begin{tabular}{|c|c|c|c|c|c|c|c|c|c|}
\hline \multirow{2}{*}{\multicolumn{2}{|c|}{$\begin{array}{c}\text { TIPO } \\
\text { DE } \\
\text { UNIÃO }\end{array}$}} & \multicolumn{3}{|c|}{ Meio anticoncepcional usado } & \multirow{2}{*}{$\begin{array}{l}\text { Já usou } \\
\text { (1) }\end{array}$} & \multirow{2}{*}{$\begin{array}{c}\text { Nunca usou } \\
\text { (2) }\end{array}$} & \multirow{2}{*}{$\begin{array}{l}\text { Não } \\
\text { responde } \\
\text { (3) }\end{array}$} & \multicolumn{2}{|c|}{ Total: $(1)+(2)+(3)$} \\
\hline & & $\begin{array}{l}\text { Pílula } \\
\text { (qualquer } \\
\text { tipo) }\end{array}$ & $\begin{array}{l}\text { Tabela } \\
\text { (Ogino- } \\
\text { Knauss) }\end{array}$ & $\begin{array}{l}\text { Operação } \\
\text { (trompas } \\
\text { ligadas) }\end{array}$ & & & & $\begin{array}{c}\mathrm{N}^{0} \\
\text { absoluto }\end{array}$ & $\%$ \\
\hline \multirow{4}{*}{\multicolumn{2}{|c|}{ 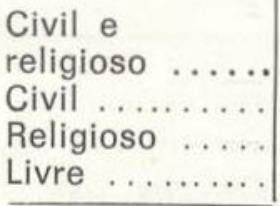 }} & & 5 & 2 & & & & & \\
\hline & & 9 & - & 3 & 12 & 13 & 2 & 27 & 28,72 \\
\hline & & 3 & - & - & 3 & 2 & - & 5 & 5,32 \\
\hline & & - & - & - & - & 2 & - & 2 & 2,13 \\
\hline \multirow{2}{*}{ Total } & $\begin{array}{l}\mathrm{N} \cdot{ }^{\circ} \text { ab- } \\
\text { soluto }\end{array}$ & 39 & 5 & 5 & 49 & 40 & 5 & 94 & - \\
\hline & $\%$ & 41,48 & 5,32 & 5,32 & 52,12 & 42,56 & 5,32 & - & 100,00 \\
\hline
\end{tabular}

OBS. - Duas das entrevistadas declararam ter usado pílula anticoncepcional antes de se submeterem à operação para esterilização definitiva, o que alterou o total geral de 92 para 94 .

TABELA vi — Ideal de filhos e idade da mulher entrevistada

\begin{tabular}{|c|c|c|c|c|c|c|c|c|c|c|c|c|c|}
\hline \multirow{2}{*}{$\begin{array}{c}\text { IDADE } \\
\text { DA MULHER } \\
\text { (em anos) }\end{array}$} & \multicolumn{11}{|c|}{ NUMERO IDEAL DE FILHOS } & \multirow{2}{*}{$\begin{array}{c}\text { SEM } \\
\text { RES- } \\
\text { POSTA }\end{array}$} & \multirow{2}{*}{ TOTAL } \\
\hline & 0 & 1 & 2 & 3 & 4 & 5 & 6 & 7 & 8 & 9 & $10+$ & & \\
\hline 15 a $19 \ldots \ldots \ldots \ldots$ & 1 & 1 & 5 & 1 & 2 & - & 1 & - & - & - & - & - & 11 \\
\hline 20 a $24 \ldots \ldots \ldots \ldots$ & 3 & 4 & 5 & 3 & 4 & 5 & - & 1 & - & - & - & - & 25 \\
\hline 25 a $29 \ldots \ldots \ldots \ldots$ & 6 & 2 & 3 & 4 & 2 & - & - & 1 & 1 & - & - & - & 19 \\
\hline 30 a $34 \ldots \ldots \ldots \ldots$ & 8 & - & 2 & 2 & 3 & 2 & 1 & 1 & 1 & - & 1 & - & 21 \\
\hline 35 a $39 \ldots \ldots \ldots \ldots$ & 3 & 1 & 1 & 5 & 1 & - & 1 & - & - & - & - & 1 & 13 \\
\hline 40 a $44 \ldots \ldots \ldots \ldots$ & 1 & - & - & - & 1 & - & - & - & - & - & - & - & 2 \\
\hline $45 \mathrm{e}+\ldots \ldots \ldots \ldots$ & - & - & - & - & - & - & - & - & 一 & - & - & - & - \\
\hline Ignorada ........... & 1 & - & 一 & - & - & - & - & - & 一 & - & 一 & - & 1 \\
\hline Total $\ldots \ldots \ldots \ldots$ & 23 & 8 & 16 & 15 & 13 & 7 & 3 & 3 & 2 & - & 1 & 1 & 92 \\
\hline
\end{tabular}


TABELA vir - Total de anos em que a mulher esteve casada ou em união livre e total de filhos que ela teve

\begin{tabular}{|c|c|c|c|c|c|c|c|c|c|c|c|c|}
\hline \multirow{2}{*}{$\begin{array}{c}\text { DURAÇÃO } \\
\text { DA } \\
\text { UNIÃO }\end{array}$} & \multicolumn{11}{|c|}{ NUMERO DE FILHOS } & \multirow{2}{*}{ TOTAL } \\
\hline & 0 & 1 & 2 & 3 & 4 & 5 & 6 & 7 & 8 & 9 & $10+$ & \\
\hline 0 a 4 & 5 & 10 & 5 & 3 & - & - & - & - & - & - & - & 23 \\
\hline 5 a 9 & - & 1 & 8 & 7 & 6 & 4 & 2 & - & 1 & - & 1 & 30 \\
\hline 10 a 14 & - & - & 2 & 9 & 1 & 4 & 4 & 3 & 1 & - & - & 24 \\
\hline $15 \mathrm{e}+$ & 1 & 1 & 1 & - & 1 & 1 & 1 & 2 & 2 & 1 & 4 & 15 \\
\hline Total & 6 & 12 & 16 & 19 & 8 & 9 & 7 & 5 & 4 & 1 & 5 & 92 \\
\hline
\end{tabular}

\section{B - Migração}

TABELA VIII - Total de anos vividos no local de nascimento, segundo as regiões - marido e mulher

\begin{tabular}{|c|c|c|c|c|c|c|c|c|c|c|}
\hline \multirow{4}{*}{$\begin{array}{l}\text { TEMPO DE } \\
\text { RESIDENCIA } \\
\text { (EM ANOS) }\end{array}$} & \multirow{4}{*}{ Cônjuge } & \multicolumn{8}{|c|}{ LOCA L DE NASCIMENTO } & \multirow{4}{*}{ TOTAL } \\
\hline & & \multicolumn{7}{|c|}{ 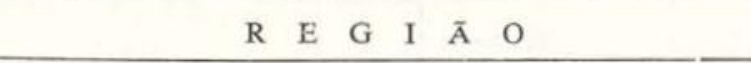 } & \multirow{3}{*}{ 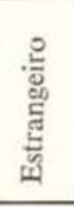 } & \\
\hline & & \multicolumn{3}{|c|}{ Norte } & \multirow{2}{*}{ 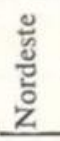 } & \multirow{2}{*}{ 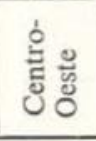 } & \multirow{2}{*}{ 㝘 } & \multirow[b]{2}{*}{$\bar{\Xi}$} & & \\
\hline & & (1) & (2) & (3) & & & & & & \\
\hline \multirow{2}{*}{ menos de $1 \ldots$} & marido & - & - & - & 1 & - & 1 & - & - & 2 \\
\hline & mulher & - & - & - & 1 & - & - & - & - & 1 \\
\hline \multirow{2}{*}{1 a $4 \ldots \ldots$} & marido & 1 & 2 & - & 2 & - & - & - & - & 5 \\
\hline & mulher & - & 1 & 1 & 1 & - & - & - & - & 3 \\
\hline \multirow{2}{*}{5 a $\quad 9 \quad \ldots \ldots$} & marido & - & 3 & 1 & - & - & 1 & 2 & - & 7 \\
\hline & mulher & 1 & 1 & 1 & 3 & - & 1 & 1 & 1 & 9 \\
\hline \multirow{2}{*}{10 a $14 \ldots \ldots$} & marido & 2 & 1 & 2 & 2 & 1 & 1 & - & - & 9 \\
\hline & mulher & 2 & 3 & 1 & 1 & - & 1 & - & - & 8 \\
\hline \multirow{2}{*}{15 a $19 \ldots \ldots$} & marido & 11 & 7 & 2 & 8 & - & 2 & 1 & - & 31 \\
\hline & mulher & 10 & 3 & 1 & 2 & - & 1 & 2 & - & 19 \\
\hline \multirow{4}{*}{$\begin{array}{l}20 \text { e }+\ldots \ldots \ldots \\
\text { desde o nasci- } \\
\text { mento até maio } \\
\text { de } 1970 \ldots \ldots \ldots\end{array}$} & marido & 4 & 2 & 4 & - & - & 3 & 1 & - & 14 \\
\hline & mulher & 8 & 3 & 5 & 5 & - & 3 & 2 & - & 26 \\
\hline & marido & 15 & - & - & - & - & - & - & - & 15 \\
\hline & mulher & 24 & - & - & - & - & - & - & - & 24 \\
\hline \multirow{2}{*}{ não sabe ....... } & marido & 3 & 3 & 2 & - & - & 1 & - & - & 9 \\
\hline & mulher & - & 1 & - & 1 & - & - & - & - & 2 \\
\hline \multirow{2}{*}{ Sub-total $=\ldots \ldots$} & marido & 36 & 18 & 11 & 13 & 1 & 9 & 4 & - & 92 \\
\hline & mulher & 45 & 12 & 9 & 14 & - & 6 & 5 & 1 & 92 \\
\hline \multicolumn{2}{|c|}{ T O T A L $\ldots \ldots \ldots \ldots \ldots$} & 81 & 30 & 20 & 27 & 1 & 15 & 9 & 1 & 182 \\
\hline
\end{tabular}


TABELA IX - Fixação de residência em diferentes localidades nos últimos 10 anos, por número de localidades - marido e mulher

\begin{tabular}{|c|c|c|c|c|c|c|c|}
\hline \multirow{3}{*}{ CONJUGE } & \multicolumn{6}{|c|}{ NUMERO DE LOCALIDADES } & \multirow{3}{*}{ TOTAL } \\
\hline & \multicolumn{2}{|c|}{1} & \multirow[t]{2}{*}{2} & \multirow[t]{2}{*}{3} & \multirow[t]{2}{*}{4} & \multirow[t]{2}{*}{$5+$} & \\
\hline & (1) & (2) & & & & & \\
\hline $\begin{array}{l}\text { Marido } \ldots . \\
\text { Mulher } \ldots\end{array}$ & $\begin{array}{l}8 \\
8\end{array}$ & $\begin{array}{l}24 \\
25\end{array}$ & $\begin{array}{l}41 \\
41\end{array}$ & $\begin{array}{l}14 \\
11\end{array}$ & $\begin{array}{l}4 \\
5\end{array}$ & $\begin{array}{l}1 \\
2\end{array}$ & $\begin{array}{l}92 \\
92\end{array}$ \\
\hline Total .... & 16 & 49 & 82 & 25 & 9 & 3 & 184 \\
\hline
\end{tabular}

(1) - Pessoas nascidas na localidade pesquisada, sem nunca ter fixado residência em outra localidade.

(2) - Demais pessoas, exceto as computadas em (1).

OBS.: Como localidades diferentes foram tomadas inclusive as vilas dentro de um mesmo Município.

TABELA $\mathrm{x}$ - Motivos alegados pelas entrevistadas que têm intenção de fixar residência em outra localidade.

\begin{tabular}{|c|c|}
\hline MOTIVACÃO PARA A MUDANCGA & $\begin{array}{l}\text { No DE ENTREVISTADAS } \\
\text { QUE DECLARARAM TER } \\
\text { INTENÇAO DE DE } \\
\text { MUDAR } \\
\text { MUDA }\end{array}$ \\
\hline $\begin{array}{l}\text { Dar melhor educação (estudo) aos filhos e procurar uma melhor situa- } \\
\text { ção financeira } \ldots \ldots \ldots \ldots \ldots \ldots \ldots \ldots \ldots \ldots \ldots \ldots \ldots \ldots \ldots \ldots \ldots \ldots \ldots \ldots \ldots \ldots \ldots \ldots \ldots\end{array}$ & 8 \\
\hline Morar perto dos pais ou outros familiares $\ldots \ldots \ldots \ldots \ldots$ & 5 \\
\hline Morar em cidade maior, com mais recursos ......... & 16 \\
\hline 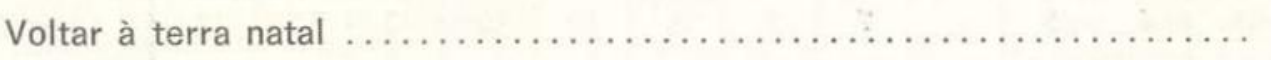 & 8 \\
\hline 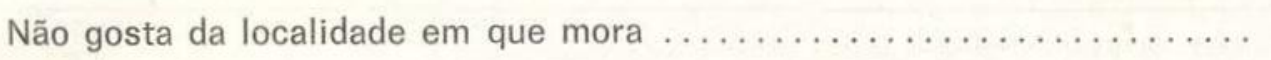 & 1 \\
\hline Por transferência do marido ........ & 26 \\
\hline 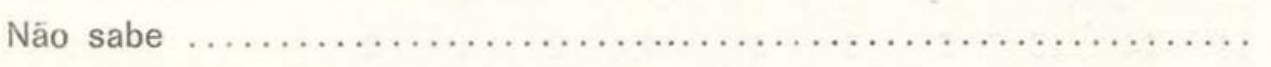 & 2 \\
\hline 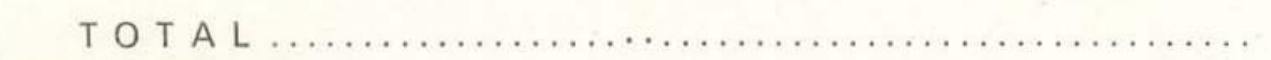 & 66 \\
\hline
\end{tabular}

C - OUTROS DADOS

TABELA XI - Filhos falecidos da mulher entrevistada e idade ao falecer

\begin{tabular}{|c|c|c|c|c|c|c|c|}
\hline $\begin{array}{l}\text { IDADE AO } \\
\text { FALECER }\end{array}$ & $\begin{array}{l}1 \text { hora a } \\
28 \text { dias }\end{array}$ & $\begin{aligned} 28 & \text { dias a } \\
3 & \text { meses }\end{aligned}$ & $\begin{array}{l}3 \text { a } 6 \\
\text { meses }\end{array}$ & $\begin{array}{l}6 \text { meses } \\
\text { a } 1 \text { ano }\end{array}$ & 1 ano & $\begin{array}{l}2 \text { anos } \\
\text { e mais }\end{array}$ & Total \\
\hline $\begin{array}{l}\text { Total de filhos } \\
\text { falecidos ..... }\end{array}$ & 10 & 3 & 4 & 11 & 5 & - & 33 \\
\hline
\end{tabular}

OBS.: - Foram registrados 3 natimortos que não estão computados na tabela. 
TABELA XII - Grau de instrução - Marido e Mulher

\begin{tabular}{|c|c|c|c|c|c|c|c|c|c|c|}
\hline \multirow[b]{2}{*}{ CONJUGE } & \multicolumn{9}{|c|}{ GRA U D E I N S TRUÇ ÃO } & \multirow[b]{2}{*}{ TOTAL } \\
\hline & 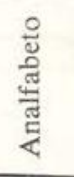 & 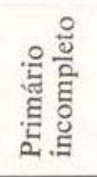 & 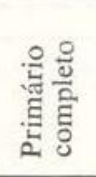 & 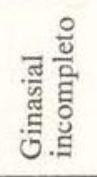 & 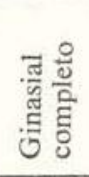 & 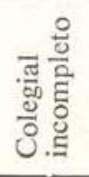 & 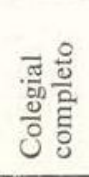 & ڤั. & 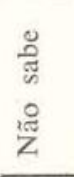 & \\
\hline $\begin{array}{l}\text { Marido } \ldots \ldots \\
\text { Mulher ........ }\end{array}$ & $\begin{array}{l}3 \\
7\end{array}$ & $\begin{array}{l}33 \\
40\end{array}$ & $\begin{array}{l}19 \\
22\end{array}$ & $\begin{array}{l}14 \\
12\end{array}$ & $\begin{array}{l}8 \\
3\end{array}$ & - & $\begin{array}{l}3 \\
6\end{array}$ & $\begin{array}{l}8 \\
1\end{array}$ & 4 & $\begin{array}{l}92 \\
92\end{array}$ \\
\hline Total $\ldots . .$. & 10 & 73 & 41 & 26 & 11 & 1 & 9 & 9 & 4 & 184 \\
\hline
\end{tabular}

TABELA XIII - Religião declarada pela entrevistada

\begin{tabular}{l|c|c|c|c|c}
\hline $\begin{array}{l}\text { RELIGIÃO } \\
\text { DECLARADA .... }\end{array}$ & Católica & Protestante & Espírita & Não responde & TOTAL \\
\hline $\begin{array}{l}\text { Total de entre- } \\
\text { vistadas ...... }\end{array}$ & 78 & 11 & 2 & 1 & 92 \\
\hline
\end{tabular}

TABELA XIV - Composição familiar e número de habitantes por domicílio

\begin{tabular}{|c|c|c|c|c|c|c|c|c|c|c|c|c|c|c|}
\hline \multirow{2}{*}{$\begin{array}{l}\text { COMPOSIÇÃO } \\
\text { DA FAMILIA }\end{array}$} & \multicolumn{13}{|c|}{ H A B I T A N TES } & \multirow{2}{*}{ TOTAL } \\
\hline & 2 & 3 & 4 & 5 & 6 & 7 & 8 & 9 & 10 & 11 & 12 & 13 & 14 & \\
\hline Casal ......... & 5 & - & - & - & - & - & 一 & - & - & 一 & - & - & - & 5 \\
\hline Casal e filhos. & - & 14 & 14 & 15 & 5 & 8 & 5 & 4 & 2 & 1 & 2 & - & 1 & 71 \\
\hline $\begin{array}{l}\text { Casal, filhos e } \\
\text { e pais ........ }\end{array}$ & - & - & 一 & - & - & - & - & - & - & 1 & 一 & - & - & 1 \\
\hline $\begin{array}{l}\text { Casal, filhos e } \\
\text { parentes ...... }\end{array}$ & - & - & 1 & 1 & 1 & 1 & 1 & - & 1 & - & - & - & - & 6 \\
\hline $\begin{array}{l}\text { Casal, filhos e } \\
\text { não parentes ... }\end{array}$ & - & - & 1 & - & 1 & 1 & 3 & - & - & - & - & - & - & 6 \\
\hline $\begin{array}{l}\text { C a s a I, filhos, } \\
\text { parentes e não } \\
\text { parentes ....... }\end{array}$ & - & - & - & - & 1 & 1 & - & - & - & - & - & - & - & 2 \\
\hline Casal e pâis .. & - & 1 & - & - & - & - & - & - & - & - & - & - & - & 1 \\
\hline TOTAL.... & 5 & 15 & 16 & 16 & 8 & 11 & 9 & 4 & 3 & 2 & 2 & - & 1 & 92 \\
\hline
\end{tabular}




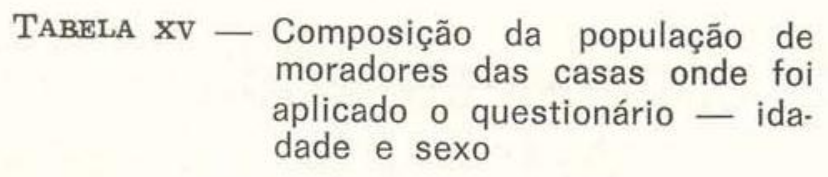

\begin{tabular}{|c|c|c|}
\hline \multirow{2}{*}{$\begin{array}{l}\text { GRUPOS } \\
\text { DE IDADE }\end{array}$} & \multicolumn{2}{|c|}{$S \quad E X O$} \\
\hline & Masculino & Feminino \\
\hline Menos de $1 \ldots$. & 14 & 14 \\
\hline 1 a $4 \ldots \ldots \ldots$ & 51 & 52 \\
\hline 5 a $9 \ldots \ldots \ldots$ & 51 & 59 \\
\hline 10 a $14 \ldots \ldots \ldots$ & 29 & 35 \\
\hline 15 a $19 \ldots \ldots$ & 11 & 30 \\
\hline 20 a $24 \ldots \ldots \ldots$ & 9 & 25 \\
\hline 25 a $29 \ldots \ldots \ldots$ & 30 & 19 \\
\hline 30 a $34 \ldots \ldots \ldots$ & 21 & 22 \\
\hline 35 a $39 \ldots \ldots \ldots$ & 21 & 13 \\
\hline 40 a $44 \ldots \ldots \ldots$ & 8 & 2 \\
\hline 45 a $49 \ldots \ldots \ldots$ & 2 & - \\
\hline 50 a $54 \ldots \ldots \ldots$ & - & - \\
\hline 55 a $59 \ldots \ldots \ldots$ & - & - \\
\hline 60 a $64 \ldots \ldots \ldots$ & - & 2 \\
\hline 65 a $69 \ldots \ldots$. & - & 1 \\
\hline $70 \mathrm{e}+\ldots \ldots \ldots$ & - & - \\
\hline Ignorada ........ & - & 1 \\
\hline Total ......... & 247 & 275 \\
\hline
\end{tabular}

\section{SUMMARY}

The Authors are studying the main population characteristics of different human groups in the rural Amazonian, region.

This paper is limited to data as collected in a military community which may not represent the average of rural Amazonian population.

Since the present data are not representative of more than $5 \%$ of the total results to be processed. the Authors have confined themselves to their presentation. Analysis and conclusions were deferred for a later date when collection will have been completed.

\section{BIBLIOGRAFIA CITADA}

Elizaga, J. C

1970 - Migraciones interiores: evolucion reciente y estado actual de dos estudios. In : Conferencia regional Latinoamericana de poblacion, México.

LEVY, M. S. F.

1969 - Catolicismo e fertilidade no distrito de São Paulo. São Paulo, Fac. de Saúde Publica da Univ. S. Paulo.

STycos, J. M.

1969 - A fertilidade humana e a América Latina. São Paulo, Ed. Univ. S. Paulo.

United Nations. Department of Social Affatres

1953a-Economic and social factors affecting fertility. In: The determinants and consequences of population trends. New York, p. 47-70.

1953b - Internal migrations. In: The determinants and consequences of population trends. New York. 